\title{
Androgen receptors are frequently expressed in mammary and extramammary Paget's disease
}

\author{
Bernadette Liegl ${ }^{1}$, Lars-Christian Horn ${ }^{2}$ and Farid Moinfar ${ }^{1}$ \\ ${ }^{1}$ Department of Pathology, Medical University of Graz, Graz, Austria and ${ }^{2}$ Department of Pathology, \\ University of Leipzig, Leipzig, Germany
}

\begin{abstract}
Mammary Paget's disease and extramammary Paget's disease are rare intraepithelial neoplasms. Mammary Paget's disease is almost exclusively associated with underlying invasive breast carcinoma or high-grade ductal carcinoma in situ (DCIS G3). Extramammary Paget's disease arises in areas rich in apocrine glands and is suspected to have apocrine gland origin. The aim of the study was to investigate the presence of estrogen receptor (ER), progesterone receptor (PR), androgen receptor (AR) and Her2/neu in a large number of cases. We investigated 58 cases of mammary and 23 cases of extramammary Paget's disease. Formalin-fixed and paraffinembedded tissues were analyzed using antibodies against AR, PR, ER and Her2/neu according to standardized procedures. In mammary Paget's disease, positive immunoreactions for Her2/neu, AR and ER were observed in 56 of $58(97 \%), 51$ of $58(88 \%)$, and respectively in six of $58(10 \%)$ cases. All cases of mammary Paget's disease were negative for PR and showed a coexpression of Her2/neu and AR in 51 out of 58 cases (88\%). In extramammary Paget's disease, positive immunoreactions for AR, Her2/neu and ER were observed in 18 of 23 (78\%), 12 of $23(52 \%)$, and respectively in 1 of $23(4 \%)$ cases. All cases of extramammary Paget's disease were negative for PR and showed a coexpression of AR and Her2/neu in 12 out of 23 cases (52\%). In contrast to ER and PR, AR and Her2/neu are commonly expressed in mammary and extramammary Paget's disease. The knowledge about frequent expression of AR in Paget's disease could lead to the development of a new adjuvant therapy, particularly in patients with recurrent disease.
\end{abstract}

Modern Pathology (2005) 18, 1283-1288. doi:10.1038/modpathol.3800437; published online 13 May 2005

Keywords: androgen receptor; breast cancer; Paget's disease; ductal intraepithelial neoplasia; ductal carcinoma in situ

Mammary Paget's disease and extramammary Paget's disease are uncommon intraepidermal epithelial neoplasms. Both conditions have similar clinical features that may mimic inflammatory and infectious skin diseases. The cells of origin in mammary Paget's disease and extramammary Paget's disease have been controversially discussed. Mammary Paget's disease is mostly associated with high-grade ductal carcinoma in situ (DCIS) (ductal intraepithelial neoplasia DIN 3) ${ }^{1}$ or invasive ductal carcinoma G2 and G3, whereas extramammary Paget's disease is associated with an underlying in situ or invasive neoplasia in up to $32 \%$ of the cases, as reported in previous studies on the basis of

Correspondence: Dr B Liegl, MD, Department of Pathology, Medical University of Graz, Auenbruggerplatz 25, 8036 Graz, Austria.

E-mail: bernadette.liegl@klinikum-graz.at

Received 24 January 2005; revised and accepted 8 April 2005; published online 13 May 2005 a small number of cases., ${ }^{2,3}$ A current theory proposes that the tumor cells of extramammary Paget's disease originate either from the intraepidermal cells of apocrine gland ducts or from pluripotent keratinocyte stem cells. ${ }^{4}$ Recently, vulvar extramammary Paget's disease has been classified based on the origin of neoplastic Paget cells as either primary (of cutaneous origin) or secondary (of non cutaneous origin). ${ }^{5}$ Mammary Paget's disease and primary extramammary Paget's disease are currently treated surgically. Mammary Paget's disease with underlying invasive ductal carcinoma or high-grade DCIS (DIN 3) is traditionally treated by mastectomy. In those cases local recurrences are rare. Recently, a more conservative surgical approach in the treatment of mammary Paget's disease is favored, particularly in cases without association to an underlying palpable tumor. ${ }^{6}$ While the conservative surgery of localized mammary Paget's disease has the major advantage of better cosmetic results, it, however, harbors a significant risk of recurrences. In 
contrast, primary vulvar Paget's disease is associated with a high recurrence rate of up to $60 \%$ after primary surgery. ${ }^{7,8}$ This relatively high rate of recurrence can be explained by either (i) positive resection margins, (ii) limitation of the vulvar surgery due to the anatomy of this region, or (iii) multifocal nature of the disease, even if the examined resection margins are histologically negative.

Normal apocrine glands of the skin and sebaceous glands are known to express androgen receptors; ${ }^{9}$ they are, however, negative for estrogen receptors (ERs) and progesterone receptors (PRs). In breast carcinomas, the role of steroid receptors in the management and treatment of patients is well established. Recently, the frequent expression of androgen receptors (ARs) in high-grade invasive breast carcinomas and in high-grade DCIS (DIN 3) has been reported. $.^{10} \mathrm{Her}-2 / \mathrm{neu}$, also known as c-erb$\mathrm{B} 2$, is another prognostic and predictive parameter used in breast carcinoma. ${ }^{11}$ The aim of this study was to investigate the expression of steroid receptors including ER, PR and AR as well as Her-2/neu on a large number of mammary Paget's disease cases and extramammary Paget's disease cases by means of immunohistochemistry.

\section{Materials and methods}

We investigated $10 \%$ buffered formalin-fixed and paraffin-embedded tissues of 81 cases of mammary Paget's disease and extramammary Paget's disease from the surgical pathology files of the Department of Pathology, Medical University Graz and from the Department of Pathology, University of Leipzig. The investigated 58 cases of mammary Paget's disease were exclusively associated with high-grade invasive ductal carcinoma (30/58) or high-grade DCIS (DIN 3, 28/58). The grading was performed by using the WHO criteria. ${ }^{1}$ None of the investigated underlying carcinomas were of apocrine type. The 23 cases of extramammary Paget's disease (21 vulva, 2 perianal) were neither associated with underlying adnexal adenocarcinomas nor internal neoplasms like anal or rectal adenocarcinomas or urothelial carcinomas. Five of them were recurrent extramammary Paget's disease cases. Paraffin-embedded tissue blocks were cut in $4 \mu \mathrm{m}$ thick serial sections and mounted on precoated glass slides. The sections were deparaffinized, rehydrated and rinsed in distilled water. Immunohistochemical assays for AR, ER, PR and Her-2/neu were performed on consecutive paraffin sections using standardized automated procedures (Ventana Medical Systems, Tucson, AZ, USA; and Dako Glostrup, Denmark) (Table 1). Monoclonal mouse antihuman antibody clones 6F11 and 1A6 (Ventana Medical Systems) were used as primary antibodies for ER and PR. For the determination of AR expression, the monoclonal mouse antihuman androgen receptor antibody (clone AR441; Dako) was used. In brief, antigen retrieval was achieved with microwave treatment (ER and PR) or heating in a water bath $\left(98^{\circ} \mathrm{C}\right)(\mathrm{AR})$ (Table 1). An ES (Ventana Medical Systems) or Chemo-Mate (Dako) autostainer was used in conjunction with an indirect streptavidin-biotin method. After incubation with the primary antibody, incubation with the secondary, biotinylated antibody was performed for $30 \mathrm{~min}$. After washing, sections were incubated with streptavidin-peroxidase for $30 \mathrm{~min}$. Finally, the enzyme was visualized after a 15-min incubation with diaminobenzidine. Counterstaining was performed with hematoxylin. Samples were only scored as positive when at least $10 \%$ of the nuclei were immunoreactive. Mammary Paget's disease and extramammary Paget's disease were also analyzed immunohistochemically with Hercep TestR, an FDA-approved assay for the detection of p185 Her2 overexpression (Dako, Denmark) (Table 1), in accordance with the manufacturer's protocol and scoring guidelines (possible score: 0 to $3+$ ). Only a score of $3+$ in more than $10 \%$ of the tumor cells was regarded as a positive result for Her-2/neu overexpression. All immunoslides were evaluated independently by two investigators (BL and FM). The rare cases, in which disagreement occurred, were re-evaluated by using a multiheaded microscope; a final agreement was reached in all cases.

Table 1 Summary of antibodies and methods in the current study

\begin{tabular}{|c|c|c|c|c|c|}
\hline Antigen & $\begin{array}{l}\text { Antibody } \\
\text { (manufacture) }\end{array}$ & Method (autostainer) & Dilution & $\begin{array}{l}\text { Incubation } \\
\text { time (min) }\end{array}$ & Antigen retrieval method \\
\hline Estrogen receptor & $\begin{array}{l}\text { 6F11 (Ventana Medical } \\
\text { Systems, Tuscon, AZ, } \\
\text { USA) }\end{array}$ & $\begin{array}{l}\text { i-view-DAB Ventana } \\
\text { (Ventana ES) }\end{array}$ & Ready to use & 32 & $\begin{array}{l}\text { Microwave at } 160 \mathrm{~W} \text { in } \\
\text { sodium citrate, } \mathrm{pH} 6.0 \text {, for } \\
30 \mathrm{~min} \text {, cooling down } 20 \mathrm{~min}\end{array}$ \\
\hline $\begin{array}{l}\text { Progesterone } \\
\text { receptor }\end{array}$ & $\begin{array}{l}\text { 1A6 (Ventana Medical } \\
\text { Systems) }\end{array}$ & $\begin{array}{l}\text { i-view-DAB Ventana } \\
\text { (Ventana ES) }\end{array}$ & Ready to use & 32 & $\begin{array}{l}\text { Microwave at } 160 \mathrm{~W} \text { in } \\
\text { sodium citrate, } \mathrm{pH} 6.0 \text {, for } \\
30 \mathrm{~min} \text {, cooling down } 20 \mathrm{~min}\end{array}$ \\
\hline Androgen receptor & $\begin{array}{l}\text { AR441 (Dako, } \\
\text { Glostrup, Denmark) }\end{array}$ & $\begin{array}{l}\text { Chem. Mate-DAB } \\
\text { (Dako Autostainer) }\end{array}$ & $1: 100$ & 20 & $\begin{array}{l}\text { Waterbath }\left(98^{\circ} \mathrm{C} \text { ) for } 40 \mathrm{~min} \text {, }\right. \\
\text { followed by cooling down } \\
20 \text { min in epitope retrieval } \\
\text { Solution (Dako) }\end{array}$ \\
\hline HER-2/neu & K5205 (Dako) Hercep T & t for the DAKO Cyton & on Autostaine & cording to & manufacturer's protocol) \\
\hline
\end{tabular}




\section{Results}

All cases of mammary Paget's disease and extramammary Paget's disease showed Paget cells with atypical round or oval nuclei and abundant basophilic, amphophilic or clear cytoplasm. The morphological appearance of the Paget cells in mammary and extramammary Paget's disease was identical. The Paget cells were present as single cells or small clusters and located throughout all layers of the epidermis (Figure 1a). Mitotic activity was variable. All cases of mammary Paget's disease were associated with an underlying high-grade DCIS (DIN 3, 28/58) or a high-grade invasive breast carcinoma (30/58). The cases of extramammary Paget's disease (21 vulvar and two perianal) were restricted to the epidermis or adnexal structures in all 23 cases. None of our cases were associated with dermal sweat gland tumors or underlying malignancy.

\section{Distribution of Steroid Receptors and Her2/neu in Mammary Paget's Disease}

ARs were positive in mammary Paget's disease in 51 out of $58(88 \%)$ cases (Figure 1d). PRs were consistently negative. ERs were negative in 52 out of 58 cases $(90 \%)$ (Figure 1b) whereas six out of $58(10 \%)$ cases of mammary Paget's disease were positive for ERs (Figure 1e). Her2/neu staining was positive in 56 out of 58 (97\%) cases (Table 2).

Co-expression of Her2/neu and AR was found in 51 out of $58(88 \%)$ cases.

\section{Distribution of Steroid Receptors and Her2/neu in Extramammary Paget's Disease}

ARs were positive in extramammary Paget's disease in 18 out of $23(78 \%)$ cases (Figure 1c). PRs were consistently negative, whereas ERs were positive in one out of $23(4 \%)$ cases. Her2/neu staining was positive in 12 out of $23(52 \%)$ cases (Figure 1f) (Table 2). Coexpression of Her2/neu and AR were found in 12 out of $23(52 \%)$ cases.

\section{Discussion}

Several previous studies on breast carcinomas revealed biological and therapeutical significance of ER and PR. ${ }^{12}$ Only a few studies examined the expression of AR in breast carcinoma. ${ }^{13}$ Moinfar et $a l^{10}$ found frequent expression of AR in poorly differentiated invasive breast carcinomas $(39 \%)$ as well as in high-grade DCIS (DIN 3, 68\%), which were mostly ER and PR negative. Few studies focused on expression of steroid hormone receptors in extramammary Paget's disease. ${ }^{14}$ These studies revealed that Paget cells in extramammary Paget's disease were usually negative for ER and PR, but commonly showed a positive immunoreaction for
AR. One study ${ }^{14}$ reported AR-positivity in 15 out of 28 extramammary Paget's disease. In this study, however, positive immunoreactivity ranged from $1 \%$ to more than $75 \%$ Paget cells. Interestingly, only eight cases $(28.5 \%)$ of extramammary Paget's disease in this study ${ }^{14}$ displayed AR-positivity in more than $10 \%$ of tumor cells.

Our study, to the best of our knowledge, is the first reporting common expression of AR in mammary Paget's disease. AR positivity in mammary Paget's disease was found in $88 \%$ of the examined cases (cutoff: positive reaction in more than $10 \%$ of Paget cells). In extramammary Paget's disease, $78 \%$ of cases revealed positive reaction for AR. While PR was always negative in mammary Paget's disease and extramammary Paget's disease, a few cases displayed ER-positivity in mammary Paget's disease $(10 \%$ of cases) and extramammary Paget's disease (4\% of cases).

Overexpression of Her2/neu was observed in mammary and extramammary Paget's disease in $96 \%$, and respectively in $53 \%$. In mammary and extramammary Paget's disease, a coexpression of Her2/neu and AR was found in $88 \%$, and respectively in $52 \%$. In $2 / 3$ of mammary Paget's disease, an underlying high-grade invasive ductal carcinoma or a high-grade DCIS (DIN 3) was present in the same sections. In the vast majority of cases, the tumor cells of invasive ductal carcinoma or DCIS (DIN) showed an identical staining pattern to that of intraepithelial Paget cells, revealing ER and PR negativity, but AR positivity. Only a minority of cases, namely two cases of DCIS G3 (DIN 3) and four cases of high-grade invasive ductal carcinoma in association with mammary Paget's disease, showed a nuclear positivity for ER. The results of Her2/neu staining of the underlying neoplasms were identical to those of the intraepidermal Paget cells in $100 \%$ of cases. The frequent expression of $\mathrm{AR}$ and common coexpression of AR and Her2/neu in mammary Paget's disease and extramammary Paget's disease raise the important question of the role of androgen, Her2/neu, and their receptors in the pathogenesis and biology of Paget's disease. Concerning breast carcinomas, a few previous studies have shown that androgens may affect the growth of breast carcinomas in animals. ${ }^{15,16}$ For example, pharmacologic administration of androgens to rats bearing dimethylbenzanthracene-induced breast carcinoma led to tumor regression. ${ }^{16}$ Tumor proliferation in human mammary carcinoma was also significantly altered by androgens. ${ }^{13}$ The results of adjuvant treatment of breast cancer with aromatase inhibitors, which block the conversion of adrenal steroids (mainly androgens) into estrogens, have been reported $^{17}$ and underscore the important role of androgens in the stimulation of human mammary carcinoma growth. Furthermore, studies on the effects of androgens and antiandrogens on breast carcinomas in long-term tissue cultures indicated that some human breast carcinomas, at least in vitro, 

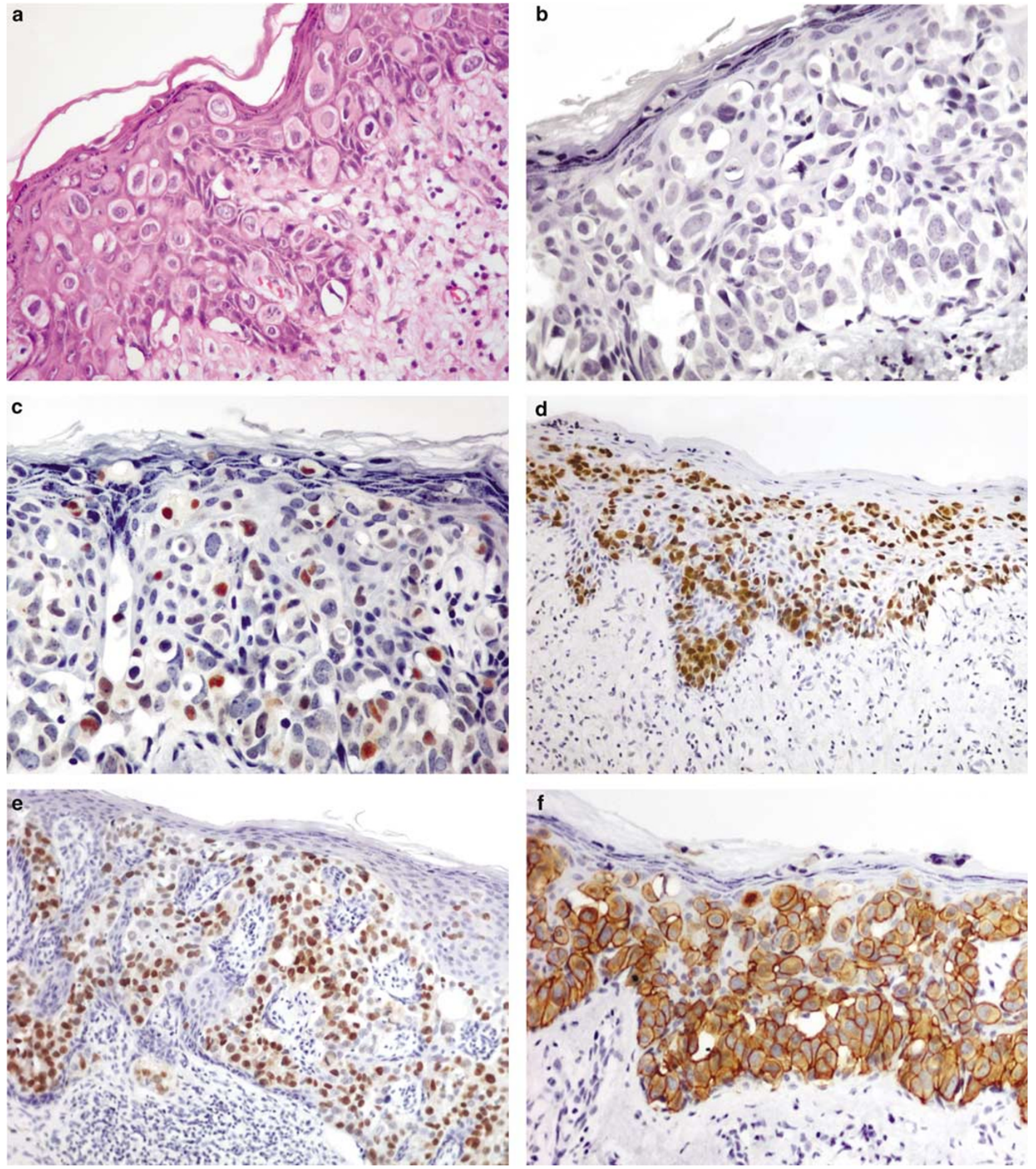

Figure 1 (a) Mammary Paget's disease showing Paget cells with atypical round or oval nuclei and abundant basophilic, amphophilic or clear cytoplasm. The Paget cells are present as single cells and located throughout all layers of the epidermis. (b) Mammary Paget's disease showing Paget cells with negative immunoreaction for ERs. (c) Vulvar extramammary Paget's disease showing Paget cells with nuclear positivity for androgen receptors in more than 10\% of tumor cells. (d) Mammary Paget's disease showing paget cells with nuclear positivity for ARs in almost $100 \%$ of tumor cells. (e) Rare example of mammary Paget's disease showing Paget cells with nuclear positivity for ERs. (f) Vulvar extramammary Paget's disease showing Paget cells with intense complete membrane staining (3+) for Her2/ neu in almost $100 \%$ of tumor cells. 
Table 2 Positive immunoreactions for AR, ER, PR and Her2/neu in mammary and extramammary Paget's disease

\begin{tabular}{lcc}
\hline & $\begin{array}{c}\text { Mammary Paget's } \\
\text { disease }\end{array}$ & $\begin{array}{c}\text { Extramammary Paget's } \\
\text { disease }\end{array}$ \\
\hline AR & $51 / 58(88 \%)$ & $18 / 23(78 \%)$ \\
ER & $6 / 58(10 \%)$ & $1 / 23(4 \%)$ \\
PR & $0 / 0(0 \%)$ & $0 / 0(0 \%)$ \\
Her2/neu & $56 / 58(97 \%)$ & $12 / 23(52 \%)$ \\
\hline
\end{tabular}

may be stimulated by androgens. ${ }^{13,15}$ On the other hand, approximately $20 \%$ of patients with metastatic breast carcinoma may experience tumor regression after treatment with androgens. ${ }^{18}$ This paradoxical affect of androgen may depend on different cell types and/or be related to the presence of other steroid receptors, such as ER or PR. In addition, the heterogeneity of carcinoma cells with respect to steroid receptor positivity and the relative distribution of each steroid receptor in carcinoma cells may influence the activity of androgens in either a proliferative or inhibitory direction. With regard to extramammary Paget's disease, an interesting experimental study ${ }^{19}$ revealed that both androgen and estrogen may stimulate the growth of a transplantable tumor derived from human extramammary Paget's disease in nude mice. Furthermore, the stimulation of growth in transplanted tumor cells in that study was clearly related to AR and ER pathways. ${ }^{19}$ Her2/neu oncogene and its relationship to breast cancer has been widely investigated $^{11,20}$ but data about its role in Paget's disease are limited. ${ }^{21-25}$ The Her2/neu oncogene is a $185 \mathrm{kDA}$ transmembrane glycoprotein with close homology to the epidermal growth factor receptor. The gene is located on chromosome 17q12-21.32. ${ }^{26}$ Overexpression of Her2/neu has been found to correlate with poor prognosis in breast cancer patients, especially node-positive ones. ${ }^{20}$ Recently, it has been shown that the spread of Paget's cells through the epidermis is induced by a motility factor that acts via the Her2/neu receptor. ${ }^{27}$ Most of the previous studies revealed a high expression of Her2/neu in mammary Paget's disease, ranging from 71 to $100 \% .^{22-24,28}$ The reported data of Her2/neu expression in extramammary Paget's disease, however, are conflicting. Previous reports have demonstrated overexpression of Her2/neu in extramammary Paget's disease ranging from 5 to $55 \% .{ }^{21-25}$ While Hannah et $a l^{21}$ found Her2/neu expression in only $5 \%$ of extramammary Paget's disease, we found Her2/neu expression in $52 \%$ of extramammary Paget's disease cases. This is similar to the findings of Takata et $a l^{25}$ who reported Her2/ neu expression in 55\% of their extramammary Paget's disease.

Currently, patients with metastatic breast carcinoma whose cancer cells overexpress Her2/neu are treated with Trastuzumab (Herceptin). Trastuzumab (Herceptin) is a recombinant DNA-derived huma- nized human monoclonal immunoglobulin G (IgG) antibody, which binds to p185 HER2. The possible antiproliferative mechanisms of Trastuzumab include downregulations of HER2 receptors, activation of immune effector cells (antibody-dependent cell-mediated cytotoxicity), reduction of S-phase cell-cycle progression, and reduction of vascular endothelial growth factor. ${ }^{29}$ Use of Trastuzumab for women suffering from recurrent vulvar Paget's disease has recently been suggested. ${ }^{30}$ It, however, needs to be emphasized that treatment with Trastuzumab (Herceptin) is associated with serious side effects, including cardiotoxicity $;^{31}$ a fact that probably limits its use as a therapeutic agent in Paget's disease. In contrast, an anti-AR treatment as currently used in the treatment of patients with prostate cancer has considerably less side effects ${ }^{32}$ and would probably be a more suitable adjuvant therapy in mammary Paget's disease and extramammary Paget's disease.

In summary, ARs are present in $88 \%$ of mammary Paget's disease and $78 \%$ of extramammary Paget's disease. Overexpression of Her2/neu can also be observed in mammary Paget's disease and extramammary Paget's disease in $96 \%$, and respectively in $52 \%$. The knowledge about frequent expression of AR in Paget's disease may play a significant role in the development of new adjuvant therapeutic strategies in future, particularly in patients with recurrent diseases.

\section{Acknowledgements}

We thank the technical staff of the Department of Pathology, Medical University Graz, especially Mrs Margit Gogg-Kammerer and Mrs Brigitte Tessaro for excellent support. We are particularly grateful to Mrs Barbara Erker and Mr Roman Christof for their excellent assistance.

\section{References}

1 Tavassoli FA, Devilee P, (eds). Pathology \& Genetics Tumours of the Breast and Femal Genital Organs. WHO Publications Center: Albany, NY, 2003.

2 Olson DJ, Fujimura M, Swanson P, et al. Immunohistochemical features of Paget's disease of the vulva with and without adenocarcinoma. Int J Gynecol Pathol 1991;10:285-295.

3 Molinie V, Paniel BJ, Lessana-Leibowitch $\mathrm{M}$, et al. Paget disease of the vulva. 36 cases. Ann Dermatol Venereol 1993;120:522-527.

4 Lloyd J, Flanagan AM. Mammary and extramammary Paget's disease. J Clin Pathol 2000;53:742-749.

5 Wilkinson EJ, Brown HM. Vulvar Paget disease of urothelial origin: a report of three cases and a proposed classification of vulvar Paget disease. Hum Pathol 2002;33:549-554.

6 Marcus E. The management of Paget's disease of the breast. Curr Treat Options Oncol 2004;5:153-160. 
7 Fanning J, Lambert HC, Hale TM. Paget's disease of the vulva: prevalence of associated vulvar adenocarcinoma, invasive Paget's disease, and recurrence after surgical excision. Am J Obstet Gynecol 1999;180:24-27.

8 Kodama S, Kaneko T, Saito M. A clinicopathologic study of 30 patients with Paget's disease of the vulva. Gynecol Oncol 1995;56:63-70.

9 Choudhry R, Hodgins M, Van der Kwast T, et al. Localization of androgen receptors in human skin by immunohistochemistry: implication for the hormonal regulation of hair growth, sebaceous glands and sweat glands. J Endocrinol 1992;133:467-475.

10 Moinfar F, Okcu M, Tsybrovskyy O, et al. Androgen receptors frequently are expressed in breast carcinomas. Cancer 2003;98:703-711.

11 Quenel N, Wafflart J, Bonichon F, et al. The prognostic value of c-erbB2 in primary breast carcinomas: a study on 942 cases. Breast Cancer Res Treat 1995;35:283-291.

12 Manni A, Arafah B, Pearson OH. Estrogen and progesterone receptors in the prediction of response of breast cancer to endocrine therapy. Cancer 1980;46: 2838-2841.

13 Lippman M, Bolan G, Huff K. The effects of androgens and antiandrogens on hormone-responsive human breast cancer in long-term tissue culture. Cancer Res 1976;36:4610-4618.

14 Diaz de Leon E, Carcangiu ML, Prieto VG, et al. Extramammary Paget disease is characterized by the consistent lack of estrogen and progesterone receptors but frequently expresses androgen receptor. Am J Clin Pathol 2000;113:572-575.

15 Smith JA, King RJ. Effects of steroids on growth of an androgen-dependent mouse mammary carcinoma in cell culture. Exp Cell Res 1972;73:351-359.

16 Luthy IA, Begin D, Labrie F. Mediation by the androgen receptor of the stimulatory and antiandrogenic actions of 17 beta-estradiol on the growth of androgen-sensitive Shionogi mammary carcinoma cells in culture. Endocrinology 1988;123:1418-1424.

17 Winer EP, Hudis C, Burstein HJ, et al. American Society of Clinical Oncology technology assessment on the use of aromatase inhibitors as adjuvant therapy for women with hormone receptor-positive breast cancer: status report 2002. J Clin Oncol 2002;20: 3317-3327.

18 AMA Committee on Research. Androgens and estrogens in the treatment of disseminated mammary carcinoma. J Am Med Assoc 1960;172:1271-1274.

19 Nishi M, Tashiro M, Yoshida H. Stimulation of growth by both androgen and estrogen of the EMP-K1 transplantable tumor with androgen and estrogen receptors from human extramammary Paget's disease in nude mice. J Natl Cancer Inst 1992;84:519-523.

20 Borg A, Tandon AK, Sigurdsson H, et al. HER-2/neu amplification predicts poor survival in node-positive breast cancer. Cancer Res 1990;50:4332-4337.

21 Hanna W, Alowami S, Malik A. The role of HER-2/neu oncogene and vimentin filaments in the production of the Paget's phenotype. Breast J 2003;9:485-490.

22 Nakamura G, Shikata N, Shoji T, et al. Immunohistochemical study of mammary and extramammary Paget's disease. Anticancer Res 1995;15:467-470.

23 Keatings L, Sinclair J, Wright C, et al. c-erbB-2 oncoprotein expression in mammary and extramammary Paget's disease: an immunohistochemical study. Histopathology 1990;17:243-247.

24 Wolber RA, Dupuis BA, Wick MR. Expression of c-erbB-2 oncoprotein in mammary and extramammary Paget's disease. Am J Clin Pathol 1991;96:243-247.

25 Takata M, Fujimoto A, Aoki $\mathrm{H}$, et al. erbB-2 overexpression but no activation of beta-Catenin gene in extramammary Paget's disease. J Invest Dermatol 1999; 113:258-262.

26 Popescu NC, King CR, Kraus MH. Localization of the human erbB-2 gene on normal and rearranged chromosomes 17 to bands q12-21.32. Genomics 1989; $4: 362-366$.

27 Schelfhout VR, Coene ED, Delaey B, et al. Pathogenesis of Paget's disease: epidermal heregulin-alpha, motility factor, and the HER receptor family. J Natl Cancer Inst 2000;92:622-628.

28 Lammie GA, Barnes DM, Millis RR, et al. An immunohistochemical study of the presence of c-erbB-2 protein in Paget's disease of the nipple. Histopathology 1989;15:505-514.

29 Carter P, Presta L, Gorman CM, et al. Humanization of an anti-p185HER2 antibody for human cancer therapy. Proc Natl Acad Sci USA 1992;89:4285-4289.

30 Reich O, Liegl B, Tamussino K, et al. p185 (HER2) overexpression and HER2 oncogene amplification in recurrent vulvar Paget's disease. Mod Pathol 2005;18: 354-357.

31 Sparano J. Cardiac toxicity of trastuzumab (Herceptin): implications for design of adjuvant trials. Semin Oncol 2001;28:20-27.

32 Denis L, Keuppens F, Smith P, et al. Maximal androgen blockade: final analysis of EORTC phase III trial 30853. EORTC Genito-Urinary Tract Cancer Cooperative Group and the EORTC Data Center. Eur Urol 1998; 33:144-151. 